\title{
Cognitive ability and paternalism
}

Gilles Saint-Paul

Université de Toulouse I, CEPR, IZA and CES-Ifo

October 7, 2002 


\begin{abstract}
This paper analyses the welfare effects of price restrictions on private contracting in a world where agents have a limited cognitive ability. People compute the costs and benefits of entering a transaction with an error. The government knows the distribution of true costs and benefits as well as that of errors. By imposing constraints on transaction prices, the government eliminates some that are on average inefficient-because the price signals that one of the parties has typically grossly overestimated its benefit from participation. This policy may increase aggregate welfare even though some of the transactions being blocked are actually efficient.

The paper also studies the extent to which the use of private consultants with sufficient intelligence by people with limited intelligence may dominate government regulation.
\end{abstract}

JEL: D81, D82, D83, H21, I3, J38, J41, J42, J8, K31, K32,

Keywords: Cognitive ability, minimum wages, regulation, price control, paternalism, intelligence 


\section{Introduction}

While economists typically believe that it is inefficient to ban a mutually advantageous transaction, in many societies one in fact observes regulations which put limits on the freedom of transaction. Such restrictions include minimum wages and limits on working hours, as well as bans on voluntary slavery and child labor; usury laws which put a cap on the interest which can be asked by a lender; rent controls and controls on the price of basic commodities; health and safety standards, etc.

Why should the law impose such restrictions, if one is to expect transactions to be profitable to both parties? Economic analysis has typically explored two main routes. First it has considered whether externalities and market failures could make such restrictions welfare-enhancing. ${ }^{1}$ Second, it has explored whether their general equilibrium effects could redistribute in favor of politically powerful social groups. ${ }^{2}$

However, if one looks at the actual, explicit motivations of such policies, they seldom refer to such effects. Rather, they are paternalistic in that they impose norms of decency and acceptability on private contracting regardless of the effect of private contracts on third parties. Minimum wages, for example, are either consider as a 'subsistence wage'-implying that there exists workers willing to work for less than their subsistence level, and that it is undesirable-or a 'social minimum' granted to all workers by the government, which refers to some notion of fairness. ${ }^{3}$

Although fairness can be embodied in people's preferences ${ }^{4}$, this is somewhat non standard and it is not clear how government intervention should look like in such a world, given that fairness concerns are already reflected in existing contracts. On the other hand, if these concerns are not reflected in

\footnotetext{
${ }^{1}$ The reader can refer, for example, to Guesnerie and Roberts (1987), Agell (1999), Agell and Lommerund (1997), Cahuc and Michel (1996).

${ }^{2}$ This is the main theme of my previous research on the political economy of labor market institutions, see Saint-Paul (2000).

${ }^{3}$ On the French minimum wage, one can refer to Conseil Supérieur de l'Emploi, des Revenus, et des Coûts (1999).

${ }^{4}$ See for example Akerlof and Yellen (1990), Kolm (1998).
} 
people's preferences, and if the externalities mentioned above are not present, then in a world populated by rational agents paternalism can only be interpreted as inefficient moralizing.

Another route, however, is to argue, as many supporters of paternalistic policies do, that people do not actually know what is good for themselves, and that the government knows better. According to that view, the government can have systematic knowledge on people's deviation from rationality, and regulate the economy accordingly. The argument rests on people being imperfectly rational, and thus unable to figure out their true benefit from entering an economic relationship. One reason is that their cognitive ability, or intelligence, is not sufficient to perform the required inferences and computations. That intelligence is limited, contrary to what is assumed in most economic models, is hardly disputable. If this were not the case, there would not be a distribution of IQ scores in the population; everybody would have the highest possible score. ${ }^{5}$

This paper provides some theoretical foundations for such an argument. It analyses the welfare effects of price restrictions on private contracting in a world where agents have a limited cognitive ability. A key ingredient is the assumption that limited cognitive ability implies that one computes the costs and benefits of entering a transaction with an error. The government does not know the true costs and benefits for a given agent, however it knows the distribution of true costs and benefits as well as that of the errors being made. This allows it to infer that transactions taking place at some prices are on average inefficient-observing them signals that one of the parties has typically grossly overestimated its benefit from participation. By banning such transactions, the government increases aggregate welfare even though some of the transactions being blocked are actually efficient. But their positive expected contribution to total welfare is smaller than the expected negative contribution of those blocked transactions which would have been inefficient.

\footnotetext{
${ }^{5}$ It is known, for example, that high IQ people perform much better in jobs with complex tasks than average IQ people, while a low IQ is a predictor of teenage crime. See American Psychological Association Task Force (1996).
} 
The paper also tackles another side of the argument, which is the following: In order for the government to be able to perform such computations, there must exist some people with sufficient cognitive ability. Instead of working for the government, these people could instead work as private consultants, i.e. sell information services to agents with low cognitive ability in order to help them to better compute the costs and benefits of transactions. If such a market for information arises and if the cost of information is low enough, it can be shown that all agents will enter transactions that are expected to be efficient, and that the scope for paternalistic price restrictions disappears. Indeed, consultants will tend to artificially boost the bargaining position of their clients beyond their true expected opportunity cost, in order to exploit monopoly power through a strategic delegation effect. Consequently, absent public intervention there are too few transactions taking place from the point of view of social welfare. Nevertheless, price restrictions need not reduce social welfare, but for a totally different reason, which is that they may reduce monopoly power (an effect well studied in the literature). Finally, government intervention may remain desirable for another motive, namely in order to exploit economies of scale in the acquisition of information.

This paper is related to a slim strand of literature regarding the implications of irrational behavior. A seminal paper by Becker (1962) introduces randomness in individual behavior, and shows that it induces restrictions on the property of the demand curve. However it does not discuss possible implications for government intervention. Akerlof and Dickens (1982) study the implications of "cognitive dissonance", i.e. the established tendency of individuals to discard information suggesting their past choices are mistaken. They provide the example of hazardous jobs, arguing that safety regulation may be Pareto-enhancing. Hence, contrary to the present paper where mistakes are random, in the Akerlof and Dickens paper the direction of mistakes is systematically predictable on the basis of observed past choices. Consequently, government intervention is truly 'paternalistic', in that the 
government does know better what is good for a given agent. In contrast, in my model paternalism only applies in a statistical sense.

The paper is organized as follows: in the next section, I set up the basic model and compute the effect of price restrictions on welfare. In section 3, I extend the model by allowing agents with limited cognitive ability to purchase consultancy services. Section 4 concludes.

\section{$2 \quad$ A simple matching model}

There is a continuum of agents of total mass 1. These agents are ex-ante identical. They match randomly with each other in order to produce $m$ units of output, where $m$ is fixed and exogenous. Each party has an opportunity cost $c$ of participating in the venture. I assume that $c$ has full support on $(-\infty,+\infty)$, being distributed with c.d.f. $G(c)$. $G$ is continuous and differentiable almost everywhere, $G^{\prime}=g$.

People do not observe their true opportunity cost. Rather, they observe it with error, believing that their cost is actually $\tilde{c}=c+\varepsilon$, where $\varepsilon$ is distributed over $(-\infty,+\infty)$ with c.d.f. $F(c)$ and density $f(c)$. Without loss of generality I assume $E(\varepsilon)=0$. Thus cognitive ability is interpreted as the size of mistakes being made regarding one's net value from participating in a match. The greater the variance of $\varepsilon$, the smaller the intelligence of people in this society.

Note that agents sincerely believe that their cost is $\tilde{c}$; they do not treat $\tilde{c}$ as a signal and do not make rational inferences on $c$ based on observing $\tilde{c}$. This is beyond their cognitive ability. People learn about their true cost only by experience, by receiving a certain level of utility after the match has produced; but it is then too late to change their mind.

Consequently, absent any government intervention, a match will actually take place if and only if it yields a positive perceived surplus. Denoting by $\tilde{c}=c+\varepsilon$ and $\tilde{c}^{\prime}=c^{\prime}+\varepsilon^{\prime}$ the perceived opportunity cost of each party, we 
get the following condition:

$$
m \geq \tilde{c}+\tilde{c}^{\prime}
$$

If that inequality is violated, then the match does not take place and each party gets a utility of zero. If not, then the parties must agree on a sharing rule prior to production taking place. Such sharing rule is observable by the government, and cannot be renegotiated. We assume it is determined by Nash bargaining, and that all agents have the same bargaining power. Consequently, the agent with a perceived opportunity cost $\tilde{c}$ (agent 1) will get a payment equal to

$$
\begin{aligned}
w & =\frac{1}{2}\left(m-\tilde{c}-\tilde{c}^{\prime}\right)+\tilde{c} \\
& =\frac{m+\left(\tilde{c}-\tilde{c}^{\prime}\right)}{2},
\end{aligned}
$$

while the other agent (agent 2) gets $w^{\prime}=\left(m-\left(\tilde{c}-\tilde{c}^{\prime}\right)\right) / 2$.

The true welfare of agent 1 is given by the difference between his wage and his opportunity cost:

$$
\begin{aligned}
\omega & =w-c \\
& =\frac{1}{2}\left(m-c-c^{\prime}\right)+\frac{1}{2}\left(\varepsilon-\varepsilon^{\prime}\right) .
\end{aligned}
$$

Not surprisingly, welfare is greater (but the match is less likely to operate), the more agent 1 has overestimated his opportunity cost. It is also greater (and the match is more likely to operate), the more agent 2 has underestimated his opportunity cost.

Given our assumptions, total social welfare is equal to the sum over all matches taking place of the match's net surplus

$$
\Omega_{0}=\iiint_{c, c^{\prime}, \varepsilon}\left(m-c-c^{\prime}\right) g(c) g\left(c^{\prime}\right) f(\varepsilon) F\left(m-c-c^{\prime}-\varepsilon\right) d c d c^{\prime} d \varepsilon .
$$




\subsection{A price restriction}

Let us now analyze how the preceding analysis is changed when the government impose a restriction on the price that can be paid on each party. I assume, and this is an important assumption, that the government knows the distribution of opportunity costs, as well as the distribution of errors, and is able to compute social welfare. Let us then consider the effects of a 'minimum wage' $\bar{w}$ on the payment to each party. Clearly, to be feasible it must satisfy $\bar{w}<m / 2$, which I shall henceforth assume.

This regulation changes both the set of matches which operate and the transfers. Specifically, one may distinguish four cases:

A. If

$$
\begin{aligned}
& \tilde{c}^{\prime}<m-\tilde{c} \\
& \tilde{c}^{\prime}<m-2 \bar{w}+\tilde{c} \\
& \tilde{c}^{\prime}>2 \bar{w}-m+\tilde{c}
\end{aligned}
$$

the match takes place and payments are unaffected by the regulation. In the above conditions, the first inequality tells us that the match is perceived as productive; the second one that agent 1's perceived opportunity cost is high enough for his negotiated payment to exceed $\bar{w}$; and the third one says that agent 2's perceived opportunity cost is high enough for his payment to exceed $\bar{w}$ too.

B. If

$$
\begin{aligned}
\tilde{c} & <\bar{w} \\
\tilde{c}^{\prime} & >m-2 \bar{w}+\tilde{c} \\
\tilde{c}^{\prime} & <m-\bar{w},
\end{aligned}
$$

the match takes place, agent 1 receives $\bar{w}$, and agent 2 receives $m-\bar{w}$. The second inequality tells us that absent the regulation, agent 1 would receive less than $\bar{w}$, due to his low perceived opportunity cost. The first one tells us that he is willing to enter the relationship if paid $\bar{w}$. The third one means 
that agent 2 is also willing to enter the relationship, i.e. it gets more than its opportunity cost after paying the minimum wage to agent 1 . Note that these two inequalities in turn imply that the match is perceived as produtive, i.e. $m \geq \tilde{c}+\tilde{c}^{\prime}$.

C. If

$$
\begin{aligned}
\tilde{c}^{\prime} & <\bar{w} \\
\tilde{c} & >m-2 \bar{w}+\tilde{c}^{\prime} \\
\tilde{c} & <m-\bar{w},
\end{aligned}
$$

we are in the symmetrical case of B. Agent 2 is paid $\bar{w}$, and agent 1 gets $m-\bar{w}$.

D. If none of these sets of inequalities hold, the match does not take place. This includes matches which would take place absent regulation, for example matches such that $\tilde{c}+\tilde{c}^{\prime}<m, \tilde{c}^{\prime}>m-2 \bar{w}+\tilde{c}$, but $\tilde{c}^{\prime}>m-\bar{w}$. In such a case agent 2 is not willing to pay $\bar{w}$ to agent 1 , although there exist lower wages that agent 1 would accept and agent 2 would be willing to pay.

The key question we are interested in is: how does such a regulation affect total social welfare? To answer that question we simply need to characterize the set of matches which actually take place. The following lemma summarizes the above taxonomy:

LEMMA 1 - A match takes place if and only

(i) $\tilde{c}<\bar{w}$ and $\tilde{c}^{\prime}<m-\bar{w}$

or (ii) $\bar{w} \leq \tilde{c} \leq m-\bar{w}$ and $\tilde{c}^{\prime}<m-\tilde{c}$

PROOF - See Appendix.

Lemma 1 is illustrated on Figure 1, which depicts the set of perceived costs which lead to a viable match (zone I) and compares it to its counterpart absent government intervention (zones I,II, and III). With this result in hand, 
we can proceed and compute social welfare by summing the net surplus of all matches which do actually take place. We get the following expression:

$$
\Omega(\bar{w})=\int_{-\infty}^{+\infty} \int_{-\infty}^{+\infty}\left(m-c-c^{\prime}\right) g(c) g\left(c^{\prime}\right) I\left(c, c^{\prime}\right) d c d c^{\prime}
$$

where $I\left(c, c^{\prime}\right)$ is given by

$$
I\left(c, c^{\prime}\right)=\int_{-\infty}^{\bar{w}-c} f(\varepsilon) F\left(m-\bar{w}-c^{\prime}\right) d \varepsilon+\int_{\bar{w}-c}^{m-\bar{w}-c} f(\varepsilon) F\left(m-c-c^{\prime}-\varepsilon\right) d \varepsilon .
$$

Clearly we have $\lim _{\bar{w} \rightarrow-\infty} \Omega(\bar{w})=\Omega_{0}$. In order to know whether a "minimum wage" may increase welfare, we first look at the marginal effect of an increase in $\bar{w}$ on welfare. Differentiating (1), we get

$\Omega^{\prime}(\bar{w})=-\iint_{c, c^{\prime}}\left(m-c-c^{\prime}\right) g(c) g\left(c^{\prime}\right)\left[f(m-\bar{w}-c) F\left(\bar{w}-c^{\prime}\right)+f\left(m-\bar{w}-c^{\prime}\right) F(\bar{w}-c)\right] d c d c^{\prime}$

Computations allow to prove the following:

PROPOSITION $1-\Omega^{\prime}(\bar{w})>0$ if and only if

$$
\frac{\int_{-\infty}^{+\infty} c g(c) f(m-\bar{w}-c) d c}{\int_{-\infty}^{+\infty} g(c) f(m-\bar{w}-c) d c}+\frac{\int_{-\infty}^{+\infty} c^{\prime} g\left(c^{\prime}\right) F\left(\bar{w}-c^{\prime}\right) d c^{\prime}}{\int_{-\infty}^{+\infty} g\left(c^{\prime}\right) F\left(\bar{w}-c^{\prime}\right) d c^{\prime}}>m .
$$

PROOF - See Appendix.

Proposition 1 gives a simple condition on the distribution of costs and misperceptions for a marginal increase in the minimum wage to increase welfare. Condition (3) has a simple Bayesian interpretation. Its left-hand side is the expected total opportunity cost of a marginal match, i.e. a match which would be destroyed if $\bar{w}$ were increased by a small amount. The first term is the mathematical expectation of the true cost of the agents who are about to give up if the minimum wage is increased. These agents have a perceived cost equal to $m-\bar{w}$, which makes it barely profitable for them to participate while paying $\bar{w}$ to their mate. By Bayes' law, the conditional 
distribution of their true opportunity cost conditional on their perceived cost being $\tilde{c}=m-\bar{w}$ is given by

$$
g(c \mid c+\varepsilon=m-\bar{w})=\frac{g(c) f(m-\bar{w}-c)}{\int_{-\infty}^{+\infty} g(c) f(m-\bar{w}-c) d c} .
$$

Consequently, the first term of (3) is $E(c \mid \tilde{c}=m-\bar{w})$. The second term is the mathematical expectation of the true opportunity cost of the marginal agents' mate. This mate is paid $\bar{w}$, and for the match to be operative it must be that $\tilde{c}^{\prime}=c^{\prime}+\varepsilon^{\prime} \leq \bar{w}$. Thus, by Bayes law the conditional distribution of $c^{\prime}$ given that this person is paid $\bar{w}$ is

$$
\frac{g\left(c^{\prime}\right) F\left(\bar{w}-c^{\prime}\right)}{\int_{-\infty}^{+\infty} g\left(c^{\prime}\right) F\left(\bar{w}-c^{\prime}\right) d c^{\prime}}
$$

so that the second term of Eq. (3) is $E(c \mid \tilde{c}<\bar{w})$.

Therefore, Equation (3) means that the expected total opportunity cost of the matches that are destroyed by an increase in the minimum wage is higher than the output of a match, so that destroying these matches overall increases social welfare. Note that some of the actual matches that will be destroyed are actually efficient. But the average contribution of destroyed matches to social welfare is negative.

If instead of a problem of limited cognitive ability, we had rational Bayesian agents who were fully aware of their misperceptions, they would correctly compute the mathematical expectation of their opportunity cost when deciding whether or not to participate in a match. Absent regulation, all existing matches would therefore satisfy the private efficiency requirement given by

$$
E(c \mid \tilde{c})+E\left(c^{\prime} \mid \tilde{c}^{\prime}\right)<m .
$$

Since the government does not observe perceived costs, but just transaction prices, its intervention cannot improve efficiency. Imposing a minimum wage could not increase welfare, because (3) could not hold, as its LHS is the 
expectation of the LHS of (4) conditional on $\tilde{c}=m-\bar{w}$ and $\tilde{c}^{\prime} \leq \bar{w}$. In other words private Bayesian agents get the maximum possible level of efficiency given their information, and the government cannot improve on it as long as it does not have more information.

On the other hand, under limited cognitive ability, people are not aware of their misperceptions and are not able to use their perceived costs and the distribution of true costs and mistakes to perform this inference process. Through its paternalistic policies, the government is performing the inference process on behalf of private agents, although it is not doing it as efficiently as if they were Bayesians, since it only observes prices. The government does not know exactly what is good for Mrs. Smith or Mr.Jones, but it can rationally infer that, given the distribution of costs and mistakes, some transactions are likely to be correlated with gross misperceptions, and therefore are on average inefficient. An increase in the 'minimum wage' will prevent these transactions from taking place and will increase welfare if (3) holds.

Note that the government would also like to promote transactions that do not take place because both parties on average overestimate their opportunity costs. However such non-existent transactions cannot be revived by the restrictions on prices that this paper analyzes. That is, a non-existent transaction does not give any signal that the government could use in order to enforce it should one expect it to be efficient. In principle, a subsidy would increase the number of transactions, but it also favors some inefficient transactions.

Equation (3) is just a local condition which does not tell us whether intervention dominates the laissez-faire. We now provide a sufficient condition for this to be true, which makes use of the asymptotic properties of the distributions of $c$ and $\varepsilon$.

We assume that these distributions satisfy the following properties:

PROPERTY (P1): $g(c)<L e^{\lambda c}$, for $c \rightarrow-\infty$

$\operatorname{PROPERTY}(\mathrm{P} 2): f(\varepsilon)>H e^{\eta \varepsilon}$, for $\varepsilon \rightarrow-\infty$ 
PROPERTY (P3): $f(\varepsilon)<F e^{-\varphi \varepsilon}$, for $\varepsilon \rightarrow+\infty$

PROPERTY (P4): $g(c)>M e^{-\mu c}$, for $c \rightarrow+\infty$.

PROPOSITION 2 - Assume properties (P1)-(P4) hold, and that

$$
\frac{\mu}{\varphi}+\frac{\eta}{\lambda}<1
$$

then there exists $w^{*}$ such that equation (3) holds for all $\bar{w}<w^{*}$. Consequently, $\Omega(\bar{w})>\Omega_{0}$ for all $\bar{w} \leq w^{*}$.

PROOF - See Appendix.

Proposition 2 provides a condition on the thickness of the tails of the distributions of $c$ and $\varepsilon$ for a binding minimum wage to increase welfare. This condition is more likely to be satisfied if:

(i) $\mu / \varphi$ is smaller, i.e. a large true opportunity cost is more likely than a large misperception, implying that people with a large perceived opportunity cost are likely to have a large true opportunity cost rather than being overpessimistic.

(ii) $\eta / \lambda$ is smaller i.e. a small true opportunity cost is less likely than a small misperception, in which case an abnormally low perceived opportunity cost is more likely to result from overoptimism than from a truly low cost.

If these conditions hold, then matches with abnormally asymmetrical payments are likely to be inefficient. The party which gets a high payment has a high perceived opportunity cost, which is probably due to a high true opportunity cost. But the party which gets a low payment has a low opportunity cost, which is likely to be due to over-optimism rather than a true low cost. Raising $\bar{w}$ destroys such matches by precluding too asymmetrical arrangements, thus contributing to overall efficiency. 


\section{$3 \quad$ A market solution?}

In the above analysis, all private agents have a limited cognitive ability, while the government is able to perform the computations necessary for eliminating matches that are on average inefficient. However, this supposes that there does exist people who are able to perform such computations, i.e. that not everybody is of limited ability. Therefore, instead of being hired by the government, these people could sell their services to the market. In particular, they could be hired as consultants by other agents in order to tell them whether they should participate in a given match and how much they should ask. One may think that if such a market arises, then consultants will perform all necessary inferences and that matches which are systematically inefficient on average will disappear.

In this section, we analyze the implications of such a market. A key difficulty is: given their limited intelligence, how will people evaluate the gains from using a consultant? Computing them rationally amounts to actually performing the consultant's job. This makes him useless and is in contradiction with our assumption of limited cognitive ability. There is no satisfactory solution to this problem, so that I will assume that the gains from using a consultant are observable, even though people are not able to compute them. We assume enough observability of such gains so as to ensure that in equilibrium, a competitive consulting market will give advices which maximize the clients' payoffs. This implies that people not only observe the payoff from using a consultant vs. not using one, but also the payoffs associated with different consulting strategies, so that in equilibrium only those which maximize their clients' utility will survive. ${ }^{6}$

I then assume that the supply of consulting services is infinitely elastic. People can pay a fixed price $\theta$ and get an advice according to the following

\footnotetext{
${ }^{6}$ To be consistent, one must in fact assume that some agents deviate by not using consultants or using inferior ones-otherwise the associated outcomes will never be observed. Let us assume that such deviations are rare and infrequent enough so as not to quantitatively affect the equilibrium.
} 
timing:

1. Pay $\theta$ to the consultant

2. Match randomly

3. Inform the consultant of the perceived cost $\tilde{c}=c+\varepsilon$

4. The consultant gives an advice in the form of a bargaining position $c^{*}$. At this stage, both the consultant and the client ignore the other party's bargaining position.

5. This bargaining position is used instead of $\tilde{c}$ to determine whether the match actually operates and the payments to each party.

Note that assumption 5 is far from neutral. I am assuming that consultants are actually able to convince their client that their outside option is $c^{*}$ rather than $\tilde{c}$, i.e. that they are able to change their preferences. This is further discussed below, but note that it is consistent with the limited intelligence assumption, since people have no means of making inferences on their true opportunity cost by themselves, and may as well trust their consultant.

In the discussion that follows, I will assume that $\theta$ is arbitrarily small, which allows to focus the analysis on the value of paternalistic policies when information is cheap enough. When this is not true, a number of additional issues arise. In particular, price restrictions affect the desirability of using a consultant, and therefore the equilibrium number of people who use a consultant.

The first question to be answered is: What is the advice that consultants will give, i.e. that which maximizes the client's expected utility? In equilibrium the other party's bargaining position, $c^{*}$, is distributed with c.d.f. $G^{*}($.$) . Contrary to the preceding analysis, G^{*}$ is now endogenous, since it is affected by the choice of those agents who use consultants.

The match takes place if and only if $c^{*}+c^{*}<m$, in which case the client gets $w=\left(m-c^{*}-c^{*}\right) / 2+c^{*}$. The agent's expected payoff is

$$
\int_{-\infty}^{m-c^{*}} d G^{*}\left(c^{\prime}\right) d c^{\prime} \int_{-\infty}^{+\infty} g(c \mid c+\varepsilon=\tilde{c})\left[\frac{m+c^{*}-c^{\prime}}{2}-c\right] d c
$$


where

$$
g(c \mid c+\varepsilon=\tilde{c})=\frac{f(\tilde{c}-c) g(c)}{\int f(\tilde{c}-c) g(c) d c}
$$

is the posterior distribution of $c$ conditional on the agent's perceived cost $\tilde{c}$, and the term in bracket is the agent's utility net of his true opportunity cost $c$.

Given our assumptions on observability of gains, consultants will set $c^{*}$ so as to maximize the expression in (6). The first-order condition is

$$
0=\frac{1}{2} G^{*}\left(m-c^{*}\right)-\frac{g^{*}\left(m-c^{*}\right)}{\int f(\tilde{c}-c) g(c) d c} \int\left(c^{*}-c\right) f(\tilde{c}-c) g(c) d c .
$$

This equation can be rewritten as

$$
c^{*}=E(c \mid c+\varepsilon=\tilde{c})+\frac{1}{2} \frac{G^{*}\left(m-c^{*}\right)}{g^{*}\left(m-c^{*}\right)}
$$

This formula tells us that the bargaining position is the sum of two effects:

A learning effect, captured by the first term which is the posterior mean of $c$ conditional on the cost signal $\tilde{c}$. This term captures the fact that consultants are performing the Bayesian learning process that agents are unable to undertake. We denote it by $\hat{c}(\tilde{c})$ and call it the 'Bayesian cost'.

A strategic delegation (or duopoly) effect, which captures the fact that people can improve their average welfare by artificially boosting their bargaining position beyond their true estimated opportunity cost. Such an effect typically arises when bargaining is delegated to a negotiator. ${ }^{7}$ In such situations it is in the interest of those who appoint the negotiator to opt for somebody whose preferences are distorted relative to their own, in order to strategically manipulate the negotiator's outside option. This allows to commit oneself to asking more than one would sincerely do, which allows to achieve some monopoly power. Here, we are assuming that agents are "convinced" by consultants to adopt $c^{*}$ as their outside option. As a result, consultants boost $c^{*}$ upwards in the interests of their clients. One may object

\footnotetext{
${ }^{7}$ See Caillaud and Rey (1995) for a survey.
} 
that agents could realize that and thus be unable to sincerely stick to $c^{*}$. But then one would have to consider either that people could not have an outside option different from $\tilde{c}$, in which case consulting simply does not work, or that they are aware that their best estimate for $c$ is $E(c \mid c+\varepsilon=\tilde{c})$, in which case their intelligence is not limited-in the sense of this paper. Here we are assuming that in some way agents are convinced by their consultants because of their limited intelligence. That is, this limitation becomes a strategic asset for consultants, who are able to use it to manipulate their client's preferences for the sake of achieving greater monopoly power. Note that the strategic delegation term is like an inverse elasticity. The ratio $g^{*}\left(m-c^{*}\right) / G^{*}\left(m-c^{*}\right)$ is the proportion of matches that the agent will lose if $c^{*}$ increases by one unit. The coefficient $1 / 2$ captures the agent's share in total surplus.

Note that given a distribution of bargaining positions $c^{*}$, Equation (8) determines the distribution of the advices $c^{*}$. An equilibrium is a distribution which is a fixed point of that transformation. Equilibrium need not exist, and need not be unique. ${ }^{8}$

Because of the strategic delegation effect, people will adopt a bargaining position which is beyond the mathematical expectation of their true opportunity cost. For all matches that actually take place, one has $m>c^{*}+c^{*}$, implying that if all matches use a consultant:

$$
m>E(c \mid c+\varepsilon=\tilde{c})+E\left(c^{\prime} \mid c^{\prime}+\varepsilon=\tilde{c}^{\prime}\right) .
$$

In other words, all operating matches are efficient conditional on the observed signals. Since there is more information in $\tilde{c}$ and $\tilde{c}^{\prime}$ than in observed transaction prices $w$ and $w^{\prime}$, the law of iterated expectations implies that $m>E\left(c \mid w, w^{\prime}\right)+E\left(c^{\prime} \mid w, w^{\prime}\right)$. Consequently, there is no match that the government would want to destroy on the basis of observed transaction prices. This is not surprising, since consultants act in the best interest of their clients, perform all the inference that can be made, and have more information than the government. A match predicted to be systematically inefficient

\footnotetext{
${ }^{8}$ As the example below makes clear, one may speculate that multiple equilibria is a pervasive feature of this problem.
} 
on average would already have been refused by one of the two parties' consultant. If anything, the government would want to subsidize matching so as to offset the strategic delegation effect, which destroys matches for which (9) holds but $m<c^{*}+c^{*}$.

Does that imply that, if people use consultants, it can never be optimal to restrict transaction prices? Not necessarily, because restrictions also affect the distribution of bargaining positions $G^{*}$. In fact, minimum wages can reduce the strategic delegation effect, since they put a floor on how much the other party can get. Hence, even though some efficient matches are destroyed, the last term of (8) is sometimes reduced, which tends to increase the number of efficient operative matches. These effects, however, have nothing to do with the agents' imperfect rationality, and would still be there as long as they can exert monopoly power; they are of the same nature as the employment-enhancing effects of minimum wages in the context of monopsony and bilateral monopoly, which are well understood. ${ }^{9}$

To illustrate these effects, consider the following simple example. While people have different perceived costs, their true opportunity cost is the same for all agents, being equal to $c<m / 2$. Consultants know it. In the absence of a minimum wage, one can show that there exists a continuum of equilibria, where a fraction $p$ of consultants advise their client to opt for a bargaining position $c^{*}=x \in[c, m / 2]$, and a fraction $1-p$ advise their clients to be "choosy" and elect $c^{*}=m-x$. For any $p \in[0,1]$, one can check that this is an equilibrium for $x=p m / 2+(1-p) c{ }^{10}$ In that equilibrium, "choosy" agents who meet other choosy agents fail to form an operative match, and this is inefficient. Since $c<m / 2$, it would be efficient if all matches operated.

\footnotetext{
${ }^{9}$ See Manning (1995, 2001), Rebitzer and Taylor (1995), Card and Krueger (1995).

${ }^{10}$ Clearly, those who claim $x$ will always end up in an operative match, while those who claim $m-x$ will only match if they meet an agent who claims $x$. Therefore, the expected net gain from strategy $x$, is equal to $V(x)=(1-p)(x-c)+p(m / 2-c)$, and for $x=$ $p m / 2+(1-p) c$ it is equal to the expected gain from strategy $m-x, v(m-x)=p(m-x-c)$. Thus consultants are indifferent between each strategy. Furthermore, any other strategy will yield a lower payoff. For $c^{*}<x$ one always matches but gets a lower payoff than for $c^{*}=x$. For $x<c^{*}<m-x$, one only matches with those who claim $x$, but gets less than for $c^{*}=m-x$. Finally, for $c^{*}>m-x$ one never matches and gets zero.
} 
Clearly, equilibria with a greater value of $p$ dominate equilibria with a lower value of $p$, and the optimum is reached at $p=1$. A minimum wage $\bar{w}>c$ eliminates all equilibria such that $p<\frac{\bar{w}-c}{m / 2-c}$. A minimum wage equal to $m / 2$ forces the economy into the efficient equilibrium where $p=1$.

Thus, introducing a market for information eliminates the role of the state as imposing a minimum standard of rationality on existing transactions. However, it introduces another problem, namely that of monopoly power, which may still require intervention.

On the other hand, if consultants are "sincere", and induce people to take their Bayesian cost as their bargaining solution, then matches take place if and only if they are efficient conditional on the available information, and the distribution of bargaining positions is not affected by government interventions. Thus they are useless, at least if informartion is cheap enough (to illustrate these ideas, a simple model is fully worked out in the Appendix).

Another issue, however, is that people may spend too much on information. Consultants all use the same information on the distribution of opportunity costs and of errors in order to formulate their advice. Greater coordination in the use of this information could be reached by government intervention. Ideally, the government could ban consulting services, compute the Bayesian cost $\hat{c}$ for any value of $\tilde{c}$, and, by publishing the results, convince people to use it in their bargain. This would reach the maximum possible level of efficiency, and in principle could be less costly that the sum of information costs when all agents use consultants. In such an ideal case, consulting is 'nationalized' and advice is given by taking into account aggregate welfare, rather than just the client's interest, which eliminates the strategic delegation bias. But, even if consultants are "sincere", i.e. abstracting from the strategic delegation bias, it can also be that aggregate welfare is larger by banning consulting and imposing the coarse price regulations (which make a less efficient use of information than consultants), than by letting the market for information operate freely. This would be so if the welfare difference (ignoring the cost of information) between the consulting 
solution and the paternalistic solution, is smaller than the difference between the total information cost of the latter and that of the former (this possibility is formally characterized in the model of the Appendix).

\section{Conclusion}

Economic theory typically assumes that agents are fully capable of computing their optimal behavior regardless of their cognitive ability and of the complexity of their environment. If so, governments cannot improve peoples' welfare by imposing constraints on them, unless there are missing markets. Yet many laws and regulations seem motivated by the view that people sometimes get fooled into arrangements which actually harm them. This paper has provided theoretical foundations for such policies, based on the government's superior ability to make statistical inferences about the true social desirability of a transaction conditional on the observed payments.

Private markets for consulting services may in principle dominate government intervention, essentially because they use information more efficiently. However, they may lead to inefficient destruction of matches because strategic delegation implies monopoly power; and another source of inefficiency is duplication of information costs. For these reasons markets do not necessarily dominate minimum wages when it comes to offsetting the mistakes of limited intelligence.

This paper perhaps leads to a reinterpretation of the notion of "fairness", which is often used to validate paternalistic policies. The 'unfair' situations that these policies avoid are transactions where one party has made a mistake, and is getting less than its true opportunity cost. Government policies can reduce the scope for such mistakes, although at the cost of suppressing similar transactions that are not mistakes.

In the model, all agents have the same cognitive ability, in that the average size of mistakes is constant across individuals. If people differed in cognitive ability, paternalistic policies would have distributional consequences; the 
most intelligent people would systematically lose from them, as they know how to compute their opportunity cost from entering a transaction with precision. 


\section{REFERENCES}

Agell, Jonas (1999), "On the benefits from rigid labour markets: Norms, market failure, and social insurance", Economic Journal and Lommerund, K.E. (1997) "Minimum wages and the incentives for skill formation", Journal of Public Economics, 64

Akerlof, George and William Dickens (1982), "The economic consequences of cognitive dissonance", American Economic Review, 72, 3, 307-319

Akerlof, George and Janet Yellen (1990) "The fair wage effort hypothesis and unemployment", Quarterly Journal of Economics, May

American Psychological Association Task Force (1996), "Intelligence: Knowns and Unknowns", American Psychologists, February

Becker, G. (1962), "Irrational Behavior and Economic Theory", Journal of Political Economy, February, 1-13.

Cahuc, Pierre and Philippe Michel (1993) "Minimum wage, unemployment and growth", European Economic Review, 40, 1996, 1463-1483

Caillaud, Bernard and Patrick Rey (1995) "Strategic aspects of delegation", European Economic Review, 39, 421-431

Card, David, and Alan B. Krueger (1995) Myth and Measurement: The New Economics of the Minimum Wage. Princeton, NJ: Princeton University Press.

Conseil supérieur de l'emploi, des revenus et des coûts (1999), Le SMIC: Salaire minimum de croissance, Paris: La Documentation Française.

Guesnerie, Roger, and Kevin Roberts (1987) "Minimum wage legislation as a second best policy", European Economic Review, 31, 490-98

Kolm, Serge-Christophe (1998), Justice and Equity: Cambridge: MIT Press

Manning, Alan (1995), "How do we know that real wages are too high ?", Quarterly Journal of Economics, 110, 1111-1125

(2001), "Monopsony and the efficiency of labour market interventions", CEP discussion paper 514, London School of Economics

Rebitzer, James and Lowell Taylor (1995) "The Consequences of Mini- 
mum Wage Laws: Some new Theoretical Ideas" Journal of Public Economics 56

Saint-Paul, Gilles (2000) The Political Economy of Labor market Institutions, Oxford: Oxford U. Press. 


\section{APPENDIX}

PROOF OF LEMMA 1 - Assume (i), or equivalently (B1) and (B3), hold. Then if (B2) holds, we are in case B and the match takes place. Assume then that $\tilde{c}^{\prime}<m-2 \bar{w}+\tilde{c}$. Then (A2) holds. Furthermore, (B1) and (B3) imply that the match is perceived as productive, i.e. that (A1) holds. Hence, if $\tilde{c}^{\prime}>2 \bar{w}-m+\tilde{c}$, case A holds. If not, then (C2) holds. Since $\tilde{c}<\bar{w}<m / 2$, (C3) also holds. Finally, we have $2 \bar{w}-m+\tilde{c}<\bar{w}$, since this is equivalent to $\tilde{c}<m-\bar{w}$; that, along with (C2), implies that (C1) holds; hence case $\mathrm{C}$ holds.

Assume (ii), or equivalently, (A1),(C3), and not-(B1). If (C2) holds, then we have $\tilde{c}^{\prime}<\tilde{c}+2 \bar{w}-m<\bar{w}$, so that (C1) and hence case $\mathrm{C}$ hold. If (C2) does not hold, (A3) holds, and one has $m-\tilde{c}<m-2 \bar{w}+\tilde{c}$, since this is equivalent to $\bar{w}<\tilde{c}$. This together with (A1) implies (A2). Consequently case A holds.

Let us now prove that if a match takes place, it satisfies (i) or (ii).

Consider case A. Assume it does not satisfy (i). Then either $\tilde{c}>\bar{w}$ or $\tilde{c}^{\prime}>m-\bar{w}$. Assume $\tilde{c}>\bar{w}$. Then for (A1) to hold it must be that $\tilde{c}^{\prime}<m-\bar{w}$. Finally, substituting (A1) into (A3) implies $\tilde{c}<m-\bar{w}$. Thus (ii) holds. Assume now $\tilde{c}^{\prime}>m-\bar{w}$. Again for (A1) to hold we must have $\tilde{c}<\bar{w}$. Plugging this into (A2) we find that $\tilde{c}^{\prime}<m-\bar{w}$, which is a contradiction.

Consider case B. Then it clearly satisfies (i), which is equivalent to (B1) and (B3).

Consider case C. Given that $\bar{w}<m / 2,(\mathrm{C} 1)$ implies that $\tilde{c}^{\prime}<m-\bar{w}$. Then either (i) holds, or $\tilde{c}>\bar{w}$. In this case, (C3) implies that the first part of (ii) holds. Since (C3) is equivalent to $\bar{w}<m-\tilde{c}$, (C1) implies $\tilde{c}^{\prime}<m-\tilde{c}$. Consequently, (ii) holds.

PROOF OF PROPOSITION 1 - By symmetry, Equation (2) can be rewritten as

$$
m I_{1}-2 I_{2}=-\Omega^{\prime}(\bar{w})
$$


, where

$$
\begin{aligned}
I_{1} & =\iint_{c, c^{\prime}} g(c) g\left(c^{\prime}\right)\left[f(m-\bar{w}-c) F\left(\bar{w}-c^{\prime}\right)+f\left(m-\bar{w}-c^{\prime}\right) F(\bar{w}-c)\right] d c d c^{\prime} \\
& =2 \iint_{c, c^{\prime}} g(c) g\left(c^{\prime}\right)\left[f(m-\bar{w}-c) F\left(\bar{w}-c^{\prime}\right)\right] d c d c^{\prime} \\
& =2\left[\int_{-\infty}^{+\infty} g(c) f(m-\bar{w}-c)\right]\left[\int_{-\infty}^{+\infty} g(c) F(\bar{w}-c)\right]>0
\end{aligned}
$$

and

$$
\begin{aligned}
I_{2}= & \iint_{c, c^{\prime}} c g(c) g\left(c^{\prime}\right)\left[f(m-\bar{w}-c) F\left(\bar{w}-c^{\prime}\right)+f\left(m-\bar{w}-c^{\prime}\right) F(\bar{w}-c)\right] d c d c^{\prime} \\
= & {\left[\int_{-\infty}^{+\infty} c g(c) f(m-\bar{w}-c)\right]\left[\int_{-\infty}^{+\infty} g(c) F(\bar{w}-c)\right] } \\
& +\left[\int_{-\infty}^{+\infty} g(c) f(m-\bar{w}-c)\right]\left[\int_{-\infty}^{+\infty} c g(c) F(\bar{w}-c)\right]
\end{aligned}
$$

Substituting into (10), and dividing by $I_{1}$, we find that $\Omega^{\prime}(\bar{w})>0$ iff (3) holds. Q.E.D.

PROOF OF PROPOSITION 2 - We show that if $\frac{\mu}{\varphi}+\frac{\eta}{\lambda}<1$, then we can construct two positive numbers $A$ and $B$, such that

$$
\begin{aligned}
\frac{\int c g(c) F(z-c) d c}{\int g(c) F(z-c) d c} \geq A z, \text { for } z \rightarrow-\infty & \\
\frac{\int c g(c) f(z-c) d c}{\int g(c) f(z-c) d c} & \geq B z, \text { for } z \rightarrow+\infty ; \\
\text { and } B & >A .
\end{aligned}
$$

Let us first construct $A$. We want the following inequality to hold for $z$ small enough:

$$
I_{1}=\int(c-A z) g(c) F(z-c)>0 .
$$

The RHS can be decomposed as

$$
\begin{aligned}
I_{1} & =\int_{-\infty}^{A z}(c-A z) g(c) F(z-c) d c+\int_{A z}^{+\infty}(c-A z) g(c) F(z-c) d c \\
& >\int_{-\infty}^{A z}(c-A z) g(c) d c+\int_{A z}^{+\infty}(c-A z) g(c) F(z-c) d c
\end{aligned}
$$


For $z$ small enough, the first term is greater than $\int_{-\infty}^{A z}(c-A z) L e^{\lambda c} d c=$ $-L e^{\lambda A z} / \lambda^{2}$. Note that integrating the inequality $f(\varepsilon)>H e^{\eta \varepsilon}$ we get that $F(\varepsilon)>H e^{\eta \varepsilon} / \eta$ for $\varepsilon$ small enough. Let $C$ such that $g(c)>M e^{-\mu c}$ for $c>C$ and $D$ such that $f(\varepsilon)>H e^{\eta \varepsilon}$, which implies $F(\varepsilon)>H e^{\eta \varepsilon} / \eta$, for $\varepsilon<D$. Then for $z$ small enough we have $A z<C$ and $z-D<C$, so that the second integral is greater than $M H \eta^{-1} \int_{C}^{+\infty}(c-A z) e^{-\mu c} e^{\eta(z-c)} d c=e^{\eta z} E$, with $E>0$.

These two inequalities in turn imply

$$
I_{1}>E e^{\eta z}-L e^{\lambda A z} / \lambda^{2}
$$

This formula implies that $I_{1}>0$ for $z$ small enough as long as

$$
A>\frac{\eta}{\lambda}
$$

Let us now construct $B$, which must satisfy

$$
\int(c-B z) g(c) f(z-c) d c>0 \text { for } z \rightarrow+\infty .
$$

A change of variable in the integral from $c$ to $z-c$ shows that this is equivalent to

$$
\int((1-B) z-x) g(z-x) f(x) d x>0 \text { for } z \rightarrow+\infty .
$$

The LHS can be decomposed as

$$
\begin{aligned}
I_{2}= & \int_{-\infty}^{(1-B) z}((1-B) z-x) g(z-x) f(x) d x \\
& +\int_{(1-B) z}^{+\infty}((1-B) z-x) g(z-x) f(x) d x
\end{aligned}
$$

Let $\bar{g}=\max _{c} g(c)$. For $z$ large enough, the last integral is greater than

$$
\bar{g} F \int_{(1-B) z}^{+\infty}((1-B) z-x) e^{-\varphi x} d x=-\frac{\bar{g} F e^{-\varphi(1-B) z}}{\varphi^{2}} .
$$


Next, for $z-C>D$ and $(1-B) z>D$, the first integral is greater than

$$
H M \int_{-\infty}^{D}((1-B) z-x) e^{-\mu(z-x)} e^{\eta x}=F e^{-\mu z}, \text { with } F>0 .
$$

Consequently, we will have $I_{2}>0$ for $z$ large enough, provided $0<B<1$ and $\mu<\varphi(1-B)$, i.e. $B<1-\mu / \varphi$. We can pick $A$ and $B$ such that $B>A$, provided $\eta / \lambda<1-\mu / \varphi$, which is equivalent to (5).

Thus, if (5) holds, $A$ and $B$ exist such that (11) hold. Consequently, for $\bar{w}$ small enough, the LHS of (3) is greater than

$$
B(m-\bar{w})+A \bar{w}=B m-(B-A) \bar{w}
$$

Given that $B>A$, this quantity is arbitrarily large for $\bar{w}$ small enough, and therefore greater than $m$. This completes the proof of Proposition 2.

\section{An example}

There are two types of opportunity cost: high, given by $c_{H}$, and low, given by $c_{L}<c_{H}$. A match between two $c_{H}$ is inefficient, i.e. $m<2 c_{H}$. With probability $p_{H}$ (resp. $p_{L}$ ), a person with opportunity cost $c_{H}$ (resp. $\left.p_{L}\right)$ makes a mistake and believes that her cost is in fact $c_{M}=\left(c_{H}+c_{L}\right) / 2$. We assume $c_{M}+c_{H}<m$, implying $c_{H}+c_{L}<m$. The proportion of $H$-types in the population is $\rho$ and matching is random. Table 1 (where $\delta=\left(c_{H}-\right.$ $\left.c_{L}\right) / 2$ ) computes all the possible matches which can take place depending on actual and perceived costs, along with the proportion of such matches in the population.

Matches of type A will fail to form, while matches of type B and C inefficiently operate.

A minimum wage strictly greater than $m-\delta$ but smaller than $m-\delta / 2$ will only eliminate type D matches, which are efficient. But a greater minimum wage may be efficient. If it is strictly greater than $m / 2-\delta / 2$ but smaller than $m / 2$, then it eliminates types B,D,E,F, and I. The total welfare value of all these matches is equal to $\Omega(B, D, E, F, I)=2 p_{H}\left(1-p_{H}\right) \rho^{2}\left(m-2 c_{H}\right)+2 \rho(1-$ 
$\rho)\left(1-p_{H} p_{L}\right)\left(m-c_{H}-c_{L}\right)+2 p_{L}\left(1-p_{L}\right)(1-\rho)^{2}\left(m-2 c_{L}\right)$. The regulation increases aggregate welfare if that quantity is negative, i.e.

$$
\begin{aligned}
p_{H}\left(1-p_{H}\right) \rho^{2}\left(2 c_{H}-m\right)> & \rho(1-\rho)\left(1-p_{H} p_{L}\right)\left(m-c_{H}-c_{L}\right) \\
& +2 p_{L}\left(1-p_{L}\right)(1-\rho)^{2}\left(m-2 c_{L}\right)
\end{aligned}
$$

\begin{tabular}{llllll}
\hline \hline Type & No. & Perc. & Prop & Surplus & Name \\
\hline HH & $\rho^{2}$ & HH & $\left(1-p_{H}\right)^{2}$ & $m-2 c_{H}<0$ & $\mathrm{~A}$ \\
& & HM & $2 p_{H}\left(1-p_{H}\right)$ & $m-2 c_{H}<0$ & $\mathrm{~B}$ \\
& & MM & $p_{H}^{2}$ & $m-2 c_{H}<0$ & $\mathrm{C}$ \\
\hline HL & $2 \rho(1-\rho)$ & $\mathrm{HL}$ & $\left(1-p_{H}\right)\left(1-p_{L}\right)$ & $m-c_{H}-c_{L}>0$ & $\mathrm{D}$ \\
& & $\mathrm{ML}$ & $p_{H}\left(1-p_{L}\right)$ & $m-c_{H}-c_{L}>0$ & $\mathrm{E}$ \\
& & $\mathrm{HM}$ & $p_{L}\left(1-p_{H}\right)$ & $m-c_{H}-c_{L}>0$ & $\mathrm{~F}$ \\
& & $\mathrm{MM}$ & $p_{H} p_{L}$ & $m-c_{H}-c_{L}>0$ & $\mathrm{G}$ \\
\hline LL & $(1-\rho)^{2}$ & $\mathrm{LL}$ & $\left(1-p_{L}\right)^{2}$ & $m-2 c_{L}>0$ & $\mathrm{H}$ \\
& & $\mathrm{ML}$ & $2 p_{L}\left(1-p_{L}\right)$ & $m-2 c_{L}>0$ & $\mathrm{I}$ \\
& & $\mathrm{MM}$ & $p_{L}^{2}$ & $m-2 c_{L}>0$ & $\mathrm{~J}$ \\
\hline \hline Table & & & &
\end{tabular}

\section{Table 1}

\begin{tabular}{lllll}
\hline \hline Type & No. & Perc. & $w_{1}$ & $w_{2}$ \\
\hline HH & $\rho^{2}$ & HH & - & - \\
& & HM & $m / 2+\delta / 2$ & $m / 2-\delta / 2$ \\
& & MM & $m / 2$ & $m / 2$ \\
\hline HL & $2 \rho(1-\rho)$ & HL & $m / 2+\delta$ & $m / 2-\delta$ \\
& & ML & $m / 2+\delta / 2$ & $m / 2-\delta / 2$ \\
& & HM & $m / 2+\delta / 2$ & $m / 2-\delta / 2$ \\
& & MM & $m / 2$ & $m / 2$ \\
\hline LL & $(1-\rho)^{2}$ & LL & $m / 2$ & $m / 2$ \\
& & ML & $m / 2+\delta / 2$ & $m / 2-\delta / 2$ \\
& & MM & $m / 2$ & $m / 2$ \\
\hline \hline
\end{tabular}

Table 1 (Cont'd).

It is straightforward to show that with a low enough cost of consulting, a "sincere" solution, where consultants induce their clients to adopt a bargaining position equal to their true expected opportunity cost conditional on their perceived opportunity cost, (i.e. the Bayesian cost), gives a higher 
welfare than this regulation, and that additional regulation imposed on that outcome only worsens welfare.

The Bayesian cost is equal to the true opportunity cost if no mistake is made, and to

$$
\hat{c}=\frac{p_{H} \rho c_{H}+(1-\rho) p_{L} c_{L}}{p_{H} \rho+(1-\rho) p_{L}}
$$

if a mistake is made.

If $\hat{c}+c_{M}<m$, then the same matches as in Table 1 take place. Furthermore this inequality is equivalent to

$$
p_{H} \rho\left(2 c_{H}-m\right)<p_{L}(1-\rho)\left(m-c_{H}-c_{L}\right),
$$

which implies that (12) is violated. Thus, while consulting is useless and would not be used, the parameter values that make it useless also make minimum wages harmful.

If $2 \hat{c}<m<\hat{c}+c_{H}$, the use of consulting destroys matches of type $\mathrm{B}$ and $\mathrm{F}$ relative to the no consulting solution. Given that a minimum wage destroys matches D,E, and I, which are efficient, in addition to B and F, consulting alone does better than the minimum wage alone. Furthermore, adding a minimum wage on top of consulting could only improve things if it destroyed matches C, i.e. if it were greater then $m / 2$ and destroyed all matches, but one can check that this is not desirable since total welfare is positive. Indeed, it is equal to $p_{H}^{2} \rho^{2}\left(m-2 c_{H}\right)+2 \rho(1-\rho)\left(1-p_{H} p_{L}\right)\left(m-c_{H}-c_{L}\right)+(1-\rho)^{2}\left(m-2 c_{L}\right)$, which, if $2 \hat{c}<m$, is greater than $2 \rho(1-\rho)\left(m-c_{H}-c_{L}\right)+\rho(1-\rho) p_{H} p_{L}\left(c_{H}-\right.$ $\left.c_{L}\right)+(1-\rho)^{2}\left(m-2 c_{L}\right)>0$.

Finally, if $m<2 \hat{c}$, the use of consulting destroys matches B,C,F,G,J. This again improves on the minimum wage solution. In both cases, $\mathrm{B}$ and $\mathrm{F}$ are eliminated. In the consulting solution, $\mathrm{C}, \mathrm{G}$, and $\mathrm{J}$ are eliminated in addition to $\mathrm{B}$ and $\mathrm{F}$, and the joint contribution of $\mathrm{C}, \mathrm{G}$, and $\mathrm{J}$ to social welfare is

$$
\begin{aligned}
& p_{H}^{2} \rho^{2}\left(m-2 c_{H}\right)+2 \rho(1-\rho) p_{H} p_{L}\left(m-c_{H}-c_{L}\right)+p_{L}^{2}(1-\rho)^{2}\left(m-2 c_{L}\right) \\
= & \left(\rho p_{H}+p_{L}(1-\rho)\right)\left[\rho p_{H}\left(m-2 c_{H}\right)+p_{L}(1-\rho)\left(m-2 c_{L}\right)\right]<0,
\end{aligned}
$$


since $m<2 \hat{c}$. Furthermore, in the minimum wage solution, D,E, and I disappear, and their contribution is positive. Thus the consulting solution clearly dominates the paternalistic one. Finally, since all matches under the consulting solution are efficient, adding a minimum wage constraint to that solution can only reduce welfare.

For non trivial information costs, the problem of excess spending on information may appear. Assume there are $N$ agents, each using a consultant who $\operatorname{costs} \theta$, and that the government could compute its optimal minimum wage by just using one consultant, i.e. spending $\theta$ once. Assume that (12) holds and that $2 \hat{c}<m<\hat{c}+c_{H}$. If the government bans consulting and imposes a minimum wage, total aggregate welfare net of information costs is equal to

$$
\begin{aligned}
& N\left[p_{H}^{2} \rho^{2}\left(m-2 c_{H}\right)+2 \rho(1-\rho)\left(p_{H} p_{L}\right)\left(m-c_{H}-c_{L}\right)\right. \\
& \left.+(1-\rho)^{2}\left(1-2 p_{L}\left(1-p_{L}\right)\right)\left(m-2 c_{L}\right)\right]-\theta
\end{aligned}
$$

If agents use consulting, then aggregate welfare is

$N\left[p_{H}^{2} \rho^{2}\left(m-2 c_{H}\right)+2 \rho(1-\rho)\left(1-\left(1-p_{H}\right) p_{L}\right)\left(m-c_{H}-c_{L}\right)+(1-\rho)^{2}\left(m-2 c_{L}\right)\right]-N \theta$.

The first policy dominates the second one iff

$\frac{N-1}{N} \theta>2 \rho(1-\rho)\left(1-p_{L}\right)\left(m-c_{H}-c_{L}\right)+2 p_{L}\left(1-p_{L}\right)(1-\rho)^{2}\left(m-2 c_{L}\right)$,

which may hold if information costs are high enough ( $\theta$ large) and if there is enough excess duplication if consulting fees ( $N$ large). 
Figure 1

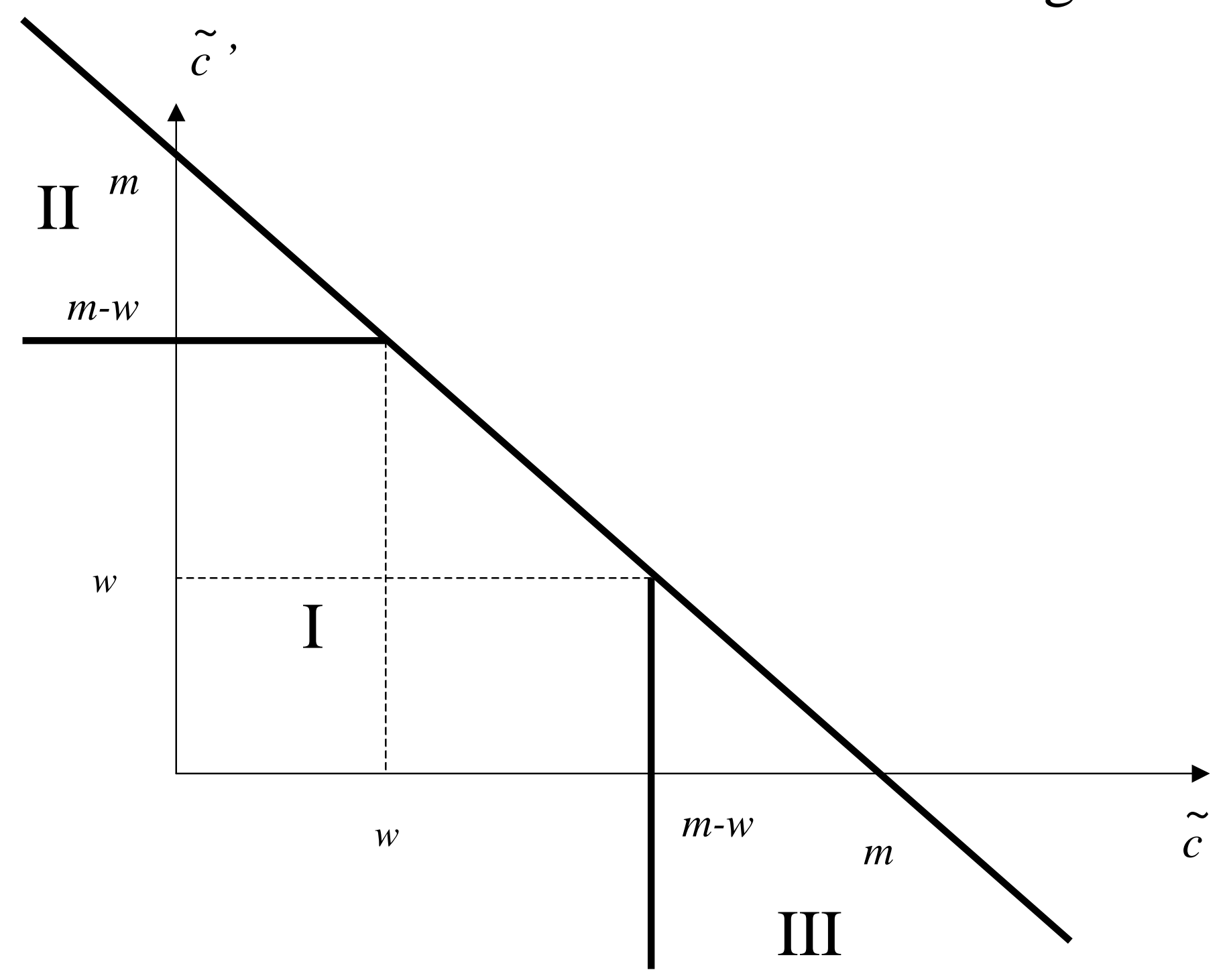

